\title{
Time series properties of the class of generalized first-order autoregressive processes with moving average errors
}

\begin{abstract}
A new class of time series models known as Generalized Autoregressive of order one with first-order moving average errors has been introduced in order to reveal some hidden features of certain time series data. The variance and autocovariance of the process is derived in order to study the behaviour of the process. It is shown that in special cases these new results reduce to the standard ARMA results. Estimation of parameters based on the Whittle procedure is discussed. We illustrate the use of this class of model by using two examples.
\end{abstract}

Keyword: Autoregression; Autocorrelations; Autocovariance; Errors; Estimation; Fractional differencing; Long memory; Moving average; Spectral density; Time series; Variance; 62M10 